\title{
I. Drei Traditionen von Wissenschaft und ihre Quellen
}

\section{Quellen moderner Wissenschaften I: Die Traditionslinie Episteme-Scientia - Der Wendepunkt im 12. Jahrhundert}

Im Zentrum des Paradigmenwechsels, der sich im 12. und 13. Jahrhundert vollzog, steht die Idee, dass Wissenschaft durch ein bestimmtes Verfahren gekennzeichnet sei, nämlich durch das Beweisen (lateinisch: demonstrare). Diese Idee ist - nicht nur zu dieser Zeit - alles andere als selbstverständlich, aber dafür umso folgenreicher. Warum prägte sie sich gerade in dieser Zeit aus?

Grund hierfür sind Verwicklungen in der Überlieferungs- und Übersetzungsgeschichte der aristotelischen Schriften. Aristotelische Texte wurden früh ins Lateinische übersetzt und das Mittelalter hindurch kontinuierlich tradiert. Dies gilt auch für die logischen Schriften aus dem sogenannten Organon - mit einer Ausnahme: die Analytica Posteriora (Zweite Analytik), die auch unter dem Titel Lehre vom Beweis geführt werden. Zwei Punkte sind hier für die Rezeption und Umsetzung in ein neues Wissenschaftskonzept entscheidend: Zum einen fand sich in diesem Werk ein Bild von Wissenschaft als einem zusammenhängendem Feld, das sich in verschiedene Bereiche unterteilt; zum anderen war dieses Vorgehen dadurch gekennzeichnet, dass es notwendige Zusammenhänge aufzeigt, also Beziehungen, für die es unmöglich ist, dass es sich anders verhält. ${ }^{1}$ Wissenschaft als eine Erkenntnisform, die sämtliche Wissensbereiche durchdringt und auf zwangsläufige

1 Vgl. Otfried Höffe: Einführung in die Wissenschaftstheorie der Zweiten Analytik. In: Aristoteles: Lehre vom Beweis oder Zweiten Analytik (Organon IV), übers.u. m. Anm. vers. v. Eugen Rolfes; m. neuer Einl. u. Bibliographie v. Otfried Höffe, Hamburg 1990, S. VII-XLII, hier XIXff. 
Weise Wahrheiten zutage fördert - das Potenzial, das hier schlummerte, liegt auf der Hand.

Dieses Verständnis einer ebenso allumfassenden wie zwingenden Vorgehensweise prägte eine wirkungsgeschichtlich mächtige und bis heute zentrale Auffassung von Wissenschaft. Wir werden noch sehen, dass diese Auffassung, trotz aller Selbstverständlichkeit, die sie in sich versammelt, keineswegs konkurrenzlos ist und dass sie auch keineswegs als eine alternativlose Deutung der aristotelischen Erkenntnislehre gelten kann. Wir werden sehen, dass der Begriff, um den sich hier alles dreht, bei Aristoteles eine ganz besondere Bedeutung hatte und eine besondere Wissensform bezeichnete. Episteme - dies ist das griechische Wort, das in den lateinischen Übersetzungen mit scientia, in modernen auch mit Wissenschaft wiedergegeben wird, ist alles andere als ein problemloser Vorläufer moderner Vorstellungen. Dazu kommt, dass Aristoteles eine Reihe anderer Erkenntnisformen kennt, die - noch bis ins 12. Jahrhundert - gleichberechtigt neben der Erkenntnisform episteme stehen, wie etwa Klugheit (phronesis) und Könnerschaft (techné). ${ }^{2}$ Letzteres, die Fertigkeit im Herbeiführen oder Herstellen, ist im lateinischen Mittelalter in ihrer Übersetzung als Kunst (ars) die Bezeichnung für das Bildungsgut, das es zu erwerben und vermitteln galt. Die Septem Artes, die Sieben freien Künste (Grammatik, Rhetorik. Dialektik, Arithmetik, Geometrie, Musik und Astronomie), gliederten die Ausbildung in den Schulen und Hochschulen, wohl geeint unter dem Dach einer sie umfassenden Theologie. ${ }^{3}$

Der wohlsituierte Status der aristotelischen episteme im Kontext eines differenzierten Feldes von Erkenntnis- und Wissensformen geriet erst ins Wanken, als sich im 12. und 13. Jahrhundert auch auf anderem Weg eine aristotelische Wissenschaftskonzeption im christlichen Abendland durchzusetzen begann. Die kastilische Stadt Toledo, 1085 erobert von den Mauren, entwickelte sich zu dieser Zeit zu einem intellektuellen Zentrum Europas. Gelehrte aus aller Welt kamen hier zusammen. Im Austausch jüdischer, christlicher und muslimischer Traditionen entstand eine einzigartige Kons-

2 Vgl. Aristoteles: Nikomachische Ethik. 6. Buch, 3-7, 1139b-1141b.

3 Vgl. Martina Roesner: Amor Scientiae und Studium generale. Die Geburt der Idee der Universität im 12./13. Jahrhundert. In: Ludger Honnefelder (Hrsg.): Albertus Magnus und der Ursprung der Universitätsidee. Die Begegnung der Wissenschaftskulturen im 13. Jahrhundert und die Entdeckung des Konzepts der Bildung durch Wissenschaft, Weilerswist 2017, S. 51-76, hier: S. 62f.; Aurelius Augustinus: Die christliche Bildung (De doctrina christiana) [426/427], Stuttgart 2002, Zweites Buch. 
tellation der Wissensvermittlung. Es wird berichtet, »der Engländer Daniel von Morley (1140-1210) [sei], gelangweilt von den einschläfernden Pariser Magistern und ihren sunerträglichen Büchern gen Toledo [aufgebrochen], um die wahre, durch die Araber vermittelte Wissenschaft zu suchen. ${ }^{4}$ Diese Wissenschaft war aber nichts anderes als eine eigenständige Überlieferungslinie der aristotelischen Schriften, verarbeitet beispielsweise von al-Fārābī (ca. 870-950) in seinem Buch Über die Wissenschaften. Dieses Buch wurde in Toledo sowohl von Gerhard von Cremona (1114-1187) als auch von Dominicus Gundissalinus (1110-1190) unter dem Titel De scientiis ins Lateinische übersetzt. Gundissalinus verarbeitete es zudem in seinem eigenständigen Werk De divisione philosophiae (Über die Einteilung der Philosophie), das im 13. Jahrhundert eine entscheidende Rolle in der »disziplinären und v.a. institutionellen Ausdifferenzierung an der Pariser Universität« spielte. ${ }^{5}$ Das entscheidende Moment dieser Traditionslinie liegt nun darin, dass sie die Breite der aristotelischen Wissensgebiete integriert zeigt und unter dem methodischen Primat der Beweislehre ordnet: Bereits al-Fārābī deutete eine Rangfolge der Wissenschaften hinsichtlich dessen an, »in wie strenger Weise die einzelnen Wissenschaften der Beweistheorie des Aristoteles entsprechen. $\ll^{6}$ Dem folgend greift Gundissalinus »die aristotelischen Überlegungen zum Habitus der Apodeixis [Beweis] als der wissenschaftlichen Haltung par excellence auf, die er zugleich differenziert in Hinblick auf die verschiedenen Beweisformen der einzelnen Wissenschaften hin interpretiert. ${ }^{7}$

Diese neue Konstellation konnte für das Verhältnis von christlicher Theologie einerseits und antiken Wissensformen und Philosophie andererseits nicht ohne Wirkung bleiben. Der umfassende Geltungsanspruch dieser

\footnotetext{
4 Alexander Fidora: Aristotelische Wissenschaft als Netzwerk von Wissenschaften. Die Rezeption der aristotelischen Wissenschaftstheorie bei al-Fārābī und Domenicus Gundissalinus. In: Ludger Honnefelder (Hrsg.): Albertus Magnus und der Ursprung der Universitätsidee. Die Begegnung der Wissenschaftskulturen im 13. Jahrhundert und die Entdeckung des Konzepts der Bildung durch Wissenschaft, Weilerswist 2017, S. 77-96, hier: S. 77.

5 Dominicus Gundissalinus: De divisione philosophiae. Über die Einteilung der Philosophie. Hrsg., übers., eingel. und mit Anm. vers. von Alexander Fidora u. Dorothée Werner, Freiburg 2007, S. 41; vgl. S. 49.

6 Franz Schupp: Einleitung. In: Al-Fārābī: Über die Wissenschaften. De scientiis. Nach der lateinischen Übersetzung Cerhards von Cremona, mit einer Einl. u. kommentierenden Anmerkungen hrsg. und übers. v. Franz Schupp, Hamburg 2005, S. XI-LXXXIV, hier S. XLI.

7 Dominicus Gundissalinus: De divisione philosophiae, Freiburg 2007, S. 29.
} 
systematischen Philosophie und ihre Gewissheit und Wahrheit verbürgende Methodologie ließ sich nicht mehr einfach in das platonisch-christliche Bild fügen, in dem die Theologie die gegenständlichen Wissensbereiche der artes als sapientia (Weisheit) überspannte. Eine Reaktion darauf war der Versuch, Teile der aristotelischen Schriften aus den Lehrplänen der Pariser Universität zu verbannen. Eine andere Strategie bestand darin, sich dieses neue Verfahren zunutze zu machen: Insbesondere in Texten aus dem späten 12. und frühen 13. Jahrhundert findet man Versuche, theologische Probleme durch teils ausdrücklich auf Aristoteles rekurrierende - Beweisverfahren zu lösen. Hintergrund sind die Auseinandersetzungen mit anderen Glaubensrichtungen und `Häresien<, die es der Theologie der Zeit geboten erscheinen ließ, neben »Mitteln göttlicher Autorität« auch "gestärkt durch die Waffen notwendiger Beweisgründe « >Irrtümer und >falsche Lehrsätze« zu bekämpfen. ${ }^{8}$

Mit der Rezeption der aristotelischen Beweis- und Wissenschaftslehre eröffnete sich die Möglichkeit, "sich auf einen Boden zu stellen, der nicht mehr primär biblisch-historischer und somit kontingenter Natur, sondern apriorisch und universalgültig ist, [...] mittels lückenloser axiomatischer Darlegung «. ${ }^{9}$ Doch das Schwert der Beweisführung war für die Theologie stets ein zweischneidiges: Wenn ein philosophisch fundiertes Verfahren die theologische Wahrheit zu sichern vermochte, dann war die übergeordnete Stellung der Theologie in ihren Grundfesten zutiefst erschüttert - eine Erschütterung, die letztlich mit einem modernen Wissenschaftsverständnis zugleich die Idee der Universität hervorbrachte - wie es treffend beschrieben wurde: Die Theologie, die die "primär texthermeneutisch orientierte Schriftauslegungstradition seit Augustinus" durch begrifflich-deduktive Vorgehensweisen ersetzt hatte, sah sich in einem zweiten Schritt gezwungen, »in ihrem deduktiven Fortschreiten früher oder später auch die Inhalte der diversen philosophischen Traktate in Betracht zu ziehen, in denen Aristoteles seine umfassende Deutung der Natur, des Menschen und der Wirklichkeit in ihrer Gesamtheit entwirft.« Die Annahme einer einheitlichen Methode führte somit - wir folgen hier den Ausführungen von Martina Rösner - dazu, »dass nun auch Gott als Gegenstand der Theologie prinzipiell mittels derselben Kategorien erkannt werden kann, die für jede andere Form

8 Mechthild Dreyer: More Mathematicorum. Rezeption und Transformation der antiken Cestalten wissenschaftlichen Wissens im 12. Jahrhundert, Münster 1996, S. 149; vgl. insgesamt S. 144-167.

9 Martina Roesner: Amor Scientiae und Studium generale. Weilerswist 2017, S. 66. 
wissenschaftlicher Gegenständlichkeit überhaupt Gültigkeit besitzen.« Und diese neue Konstellation schlägt sich auch institutionell nieder:

»Die Tatsache, dass selbst in der christlichen Theologie keine grundlegend andere facultas cognoscendi zum Einsatz kommt als in den übrigen Wissenschaften, ist somit die ideelle Voraussetzung dafür, dass auch im Rahmen der Universität die im institutionellen Sinne verstandene facultas theologica nicht über, sondern neben den anderen Fakultäten zu stehen kommt und nur noch im Rahmen des ihr eigenen Cegenstandsgebiets, nicht jedoch im Hinblick auf die anderen wissenschaftlichen Disziplinen eine Urteilskompetenz in Bezug auf die Relevanz und Cültigkeit des jeweiligen wissenschaftlichen Diskurses besitzt«. ${ }^{10}$

\section{Episteme: Der Anfang von Wissenschaft?}

Doch halten wir kurz inne - wovon sprechen wir? Es geht uns um Wissenschaft(en) in unserer gegenwärtigen Situation. Um diese im Ansatz zu verstehen, haben wir einen Blick geworfen in die Zeit um 1200 als einer zentralen Umbruchsphase. Es findet sich zwar schon in dieser Zeit ein mittelhochdeutsches Wort wizzen(t)schaft, das ganz allgemein »Wissen, Kenntnis, Kunde « bezeichnete. ${ }^{11}$ Der Diskurs um 1200 wurde jedoch nicht deutschsprachig, sondern lateinisch geführt. Vokabeln wie doctrina (Lehre), artes (Künste) und disciplina spielten hier eine Rolle. Geprägt wurde die Transformation um 1200 aber vom Begriff der scientia ${ }^{12}$, ein Wort, das sich im Englischen und den romanischen Sprachen als science, cientia, ciencia, szientia erhalten hat. Scientia wiederum, das vom Verb scire (wissen; etymologisch von scheiden, unterscheiden) her gebildete Substantiv, diente schon in der Antike zur Übersetzung der griechischen Vokabel episteme. Als nun mit der Übersetzung der Zweiten Analytik die episteme in einer schärferen und

10 Martina Roesner: Amor Scientiae und Studium generale, Weilerswist 2017, S. $75 f$.

11 Siehe Eintrag >Wissen in: Etymologisches Wörterbuch des Deutschen. Q - Z; erarbeitet von einem Autorenkollektiv des Zentralinstituts für Sprachwissenschaft und der Leitung von Wolfgang Pfeifer, Berlin 1989, S. 1984.

12 Vgl. hierzu den Eintrag /Wissenschaft< in: Historisches Wörterbuch der Philosophie, hrsg. v. Joachim Ritter, Bd. 12, S. 902-948, hier insbes. S. $903 \mathrm{ff}$. 
spezifischeren Bedeutung erschien, da prägte sich auch scientia in diesem Sinne aus. Heißt dies jedoch, dass moderne Wissenschaft auf scientia und scientia auf episteme zurückgeht?

In der Wissenschaftsphilosophie und Philosophie der Antike wird dies als selbstverständlich angenommen und fraglos reproduziert. Episteme wird - häufig recht inkonsequent - ins Deutsche mal als Wissen, mal als Wissenschaft übersetzt. Wo es als Wissenschaft übersetzt und verhandelt wird, scheint es zuweilen so, als ob alle Bereiche geistiger Tätigkeit als Wissenschaft gelten, meist aber, dass nur speziell jene Erkenntnis, die zu sicherem Wissen notwendiger Verhältnisse führt, mit diesem Begriff zu bezeichnen sei. Aristoteles hatte sich hier viel klarer ausgedrückt als seine Interpreten: Diese nämlich ergänzen bereits in den Übersetzungen das Wort Wissenschaft an Stellen, an denen es im griechischen Text gar nicht steht und übersetzen zudem ganz unterschiedliche aristotelische Begriffe mit Wissen und Wissen-

\section{BASISWISSEN 1: Episteme im Kontext}

Episteme ist das griechische Wort, das ins Lateinische mit scientia und im deutschen mit den Vokabeln Wissenschaft und Wissen, in die romanischen Sprachen und ins Englische mit science, cientia, ciencia, szientia übersetzt wird. In diesen Übertragungen spiegelt sich eine Traditionslinie der Auffassung von Wissenschaftlichkeit wider, die Wissenschaft mit logischer Beweisführung (apodeixis; demonstratio) identifiziert. Um die Komplexität dieses Überlieferungsgeschehens zu verstehen, ist es allerdings wichtig, sich die Eigenständigkeit und Besonderheit des griechischen Konzepts vor Augen zu führen. Eine Passage aus der Nikomachischen Ethik (einem späten Werk des Aristoteles), macht den Gesamtzusammenhang klar, wie er in die abendländische Tradition eingegangen ist:

Fünf geistige Bereiche seien es, die dem Menschen Wahrheit erschließen: »Kunst [techné], Wissenschaft [episteme], Klugheit [phronesis], Weisheit [sophia] und Verstand [nous] «. Bereits in der Wortstellung zeigt sich in dieser Eröffnung episteme als ein Vermögen unter anderen. Und zwar:

»Was die Wissenschaft [episteme] sei, erhellt, wenn wir die Worte genau nehmen [...], aus folgendem. Wir alle halten dafür, daß das, was man weiß, sich nicht anders verhalten kann, was sich aber anders verhalten kann, von dem weiß man, sobald man es nicht mehr vor Augen hat, nicht, 
schaft. Dies hat den Effekt, dass bereits in die Übersetzungen an vielen Stellen sich moderne Vorstellungen von Wissenschaft eingeschlichen haben, die sich erst hunderte von Jahren später allmählich ausbildeten.

Episteme als ein bestimmter Ausschnitt der Erkenntnisformen des Menschen ist aber noch in anderer Hinsicht etwas ganz anderes als Wissenschaft im modernen Sinne: Sie umfasst nämlich weder die Erfahrung (empeiría), auf der sie gleichwohl basiert, noch das Wissen um die Prinzipien (arché), von denen sie in der Beweisführung ausgeht. Beides findet sich in modernen Vorstellungen von Wissenschaft aber unbedingt integriert. Zu beiden Punkten später mehr. Die Überzeugung, dass es eine einzige Wissensform gibt, die der einzige und alleinige Weg zu wahrer Erkenntnis ist - diese für die Moderne typische Vorstellung - ist also jedenfalls nicht aristotelisch. Unsere heutige Situation ist viel enger an die Entwicklungen um 1200 gebunden als an den aristotelischen Begriff der epistéme.

ob es noch ist oder nicht. Mithin ist, was Gegenstand des Wissens ist, aus Notwendigkeit. Mithin ist es ewig; denn alles, was schlechthin aus Notwendigkeit ist, ist ewig, das Ewige aber ist ungeworden und unvergänglich.« (Aristoteles, Nikomachische Ethik, 6. Buch, 3.2, 1139b, 18ff.)

Und weiter:

»Was sich anders verhalten kann, ist teils Gegenstand des Herstellens [poiesis], teils Gegenstand des Handelns [praxis]«. Klugheit im Handeln und Kunstfertigkeit im Hervorbringen stehen gemäß dieser Konzeption als durch >Vernunft‘ geleitete - wörtlicher wäre: logos-basierte - Haltungen [hexis] auf gleicher Stufe neben der episteme, die ihrerseits einen bestimmten Umgang mit dem logos pflegt.

Für Aristoteles steht also episteme neben anderen Bereichen von Erkenntnis und Vernunft im menschlichen Leben. Wissen, in unserem heutigen Gebrauch des Wortes, findet sich in allen diesen Bereichen, episteme ist dagegen aus der Perspektive unseres heutigen Sprachgebrauchs eine sehr spezielle Form von Wissen, ein Wissen von Gegenständen, unter der Voraussetzung, dass sie nicht anders sein können. Wer Wissenschaft in dieser Traditionslinie betrachtet, impliziert auch ein solches Bild von Erkenntnisgegenständen als notwendige und zeitlose Gegebenheiten (sub specie aeternitatis - in der Gattung des Ewigen (Spinoza)). 


\section{Die Verallgemeinerung der Episteme: Wissenschaft als Beweisen}

Nimmt man ein beliebiges wissenschaftsphilosophisches Studienbuch der Gegenwart zur Hand und schlägt nach, was unter einer wissenschaftlichen Erklärung zu verstehen sei, so trifft man üblicherweise auf eine solche Darstellung: ${ }^{13}$

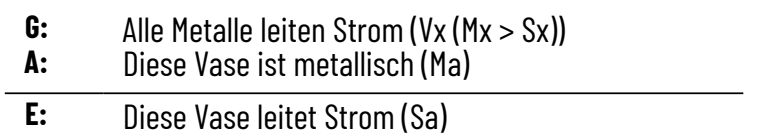

Die so darstellbare Form der wissenschaftlichen Erklärung wird nach Carl Gustav Hempel und Paul Oppenheim, die 1948 einen seitdem tausende Mal zitierten Text zu diesem Thema verfassten, das HO-Schema oder auch das Deduktiv-Nomologische (DN) Modell der Erklärung genannt. Die Gründe, warum dieser Ansatz in der Mitte des 20. Jahrhunderts eine solche Wirkkraft entfalten konnte, liegen in der speziellen Konstellation der Zeit. Sie liegen nicht darin, dass hier etwas bahnbrechend Neues gesagt wäre. Tatsächlich nämlich reproduziert das DN-Modell nichts anderes als das Verfahren der apodeixis, wie es Aristoteles in der Zweiten Analytik ausführlich darstellt. So analysiert etwa Aristoteles das Phänomen, dass Planeten nicht funkeln, so: »es sei etwa C Planeten, B das Nahesein, das A das Nicht-Funkeln; so trifft also das B [Nahesein] auf das C [Planeten] zu und das A [Nicht-Funkeln] auf das B [Nahesein], so dass auch das $C$ auf das A zutrifft [dass also Planeten nicht funkeln] ${ }^{14}$ Das Prinzip, dass Lichter, die nahe sind, nicht funkeln, vermittelt zwischen den beobachteten Planeten und der Aussage, dass sie nicht funkeln, in der Weise, dass die allgemeine Regel die Ursache dafür liefert, warum sie nicht funkeln: weil sie nahe sind. Ganz ähnlich wird die Mondfinsternis auf die Stellung der Erde und auf die Regel, dass Licht durch Gegenstände verringert werden kann, zurückgeführt..$^{15}$

13 Gerhard Schurz: Wissenschaftliche Erklärung. In: Wissenschaftstheorie. Ein Studienbuch, hrsg. v. Andreas Bartels u. Manfred Stöckler, Paderborn 2007, S. 69-88, hier S. 72.

14 Aristoteles: Lehre vom Beweis oder Zweiten Analytik (Organon IV), übers., mit einer Einl. u. Anm. hrsg. v. Wolfgang Detel, Hamburg 2011, Buch I, 13, 78b.

15 Aristoteles: Lehre vom Beweis oder Zweiten Analytik, Buch II, 2, 90 a. 
Es ist angemerkt worden, dass die Wiederentdeckung der Idee der aristotelischen Beweislehre »in der analytischen Philosophie des 20. Jahrhunderts [nur] aufgrund historischer Ignoranz als bedeutende Innovation gefeiert « werden konnte. ${ }^{16}$ Andere vertreten die Ansicht, das DN-Modell sei als eine genauere Ausführung der aristotelischen Überlegungen zu betrachten. ${ }^{17}$ Doch es bleibt dabei: die Grundfigur des Verfahrens, das heute erklären heißt, ist das aristotelische Beweisen, die apodeixis. Bis in die Gegenwart ist der Zusammenschluss von apodeixis-erklären-Wissenschaft in weiten Bereichen der Wissenschaftsphilosophie schlicht vorausgesetzt: »Die Entdeckung der Möglichkeit von Beweis ist zugleich die Entdeckung der Möglichkeit von Wissenschaft «, liest man etwa in einem einschlägigen Lexikonartikel. ${ }^{18}$

Philosophiegeschichtlich lässt sich diese bemerkenswerte Kontinuität gut nachvollziehen. Ausgehend von den Interpretationen der Zweiten Analytik im 13. Jahrhundert wurde das Motiv des Erschließens von Wirklichkeit mittels logischer Ableitung zum Standard dessen, was an Universitäten unter scientia verstanden wurde. Die im 13. Jahrhundert etablierte Vorstellung von Wissenschaften als axiomatisch-deduktive Systeme findet sich wieder bei Petrus Ramus (1543) und René Descartes, der in seinen Regulae ad directionem ingenii (vor 1628) schreibt, dass Wissenschaft aus Prinzipienkenntnis und Deduktion bestehe; Deduktion verstanden als Erkenntnis, die "aus anderem sicher Erkannten mit Notwendigkeit erschlossen wird. ${ }^{19}$ Thomas Hobbes

16 Wolfgang Detel: >Einleitung`zu: Aristoteles: Lehre vom Beweis oder Zweite Analytik, 2011, S. XXXII, Fn. 46.

17 Vgl. Schurz: >Wissenschaftliche Erklärung`2007, S. 69.

18 Vgl. hierzu den Eintrag >Beweis in: Historisches Wörterbuch der Philosophie, hrsg. v. Joachim Ritter, Bd. 1, S. 882-886, hier S. 885.

19 Descartes anerkennt nur zwei Formen von Erkenntnis: Prinzipienerkenntnis durch Intuition und Deduktion; zum Verhältnis der beiden gibt folgende Textstelle Aufschluss: »weshalb wir hier außer der Intuition noch eine andere Art der Erkenntnis angefügt haben, die durch Deduktion geschieht, worunter wir all das verstehen, was aus etwas anderem sicher Erkannten mit Notwendigkeit erschlossen wird. Aber das musste so geschehen, weil das meiste zuverlässig gewußt wird, obgleich es selbst nicht evident ist, wofern es nur aus wahren und erkannten Prinzipien durch eine zusammenhängende und nirgendwo unterbrochene Tätigkeit des Denkens, welches das einzelne deutlich in der Intuition sieht, deduziert ist, nicht anders als wenn wir das letzte Glied einer langen Kette mit dem ersten zusammenhängend erkennen, obgleich unsere Augen nicht mit einem und demselben Blick auf alle Zwischenglieder, von denen jener Zusammenhang herrührt, achten, wenn sie sie nur alle eins nach dem anderen durchmustert haben, und 
bezeichnet (1651) Wissenschaft als Verknüpfung »zu Syllogismen, den Verbindungen einer Behauptung mit einer anderen, bis wir alles kennen«, und stellt klar: »Und eben dies nennt man Wissenschaft. $\aleph^{20}$ Das Konzept einer deduktiv verfahrenden Wissenschaft zieht sich, mit einigen Schwankungen, durch bis in die Philosophie des 20. Jahrhunderts, in dessen logisch-empiristischer Tradition das DN-Modell wissenschaftlichen Erklärens steht.

Doch was hat es mit der Idee von Wissenschaft als Beweistätigkeit auf sich? Im Kanon der Wissenschaftsphilosophie ist die Verknüpfung von Wissenschaft und Beweisen so omnipräsent und selbstverständlich, dass es fast unmöglich scheint, Wissenschaft in irgendeiner Weise anders zu denken. Und doch zeigen sich Bruchlinien in diesem Gefüge, sobald man etwas näher hinsieht. Diese Bruchlinien haben mit dem Verhältnis von Logik und Mathematik ebenso zu tun wie mit den Begriffen Erfahrung, Empirie und Experiment. Auf diese einschneidenden, aber nicht ganz einfach zu sortierenden Unterschiede kommen wir später zu sprechen. Ein Unterschied aber scheint ganz deutlich und unproblematisch zu sein. Wir kennen ihn z.B. schon aus der Einteilung von Schulen und Fächern und es sieht so aus, als würde er ganz natürlich eine Einteilung der Welt der Wissenschaften in zwei Bereiche abbilden: den Bereich der Natur- und den Bereich der Geisteswissenschaften. In den Naturwissenschaften wird erklärt, so heißt es, und in den Geisteswissenschaften verstanden. Erklären und Verstehen: zwei Verfahren, die auf zwei Gattungen von Gegenständen abgestellt zu sein scheinen. Ganz einfach - aber ist das wirklich so natürlich und einfach? Was hat es mit dieser Zweiteilung auf sich? Wie kam es dazu?

wir uns erinnern, daß die einzelnen Clieder mit ihren Nachbargliedern vom ersten bis zum letzten zusammenhängen. «Intuitio bestimmt Descartes offensichtlich im Anschluss an das antik griechische Konzept des geistigen Erfassens, nous (vgl. Kurt von Fritz: Die Rolle des Noein. Um die Begriffswelt derVorsokratiker, Darmstadt 1968. Siehe auch o. BASISWISSEN 1: Episteme im Kontext): »Unter Intuition verstehe ich nicht das schwankende Zeugnis der sinnlichen Wahrnehmung oder das trügerische Urteil der verkehrt verbindenden Einbildungskraft, sondern ein so müheloses und deutlich bestimmtes Begreifen des reinen und aufmerksamen Geistes, daß über das, was wir erkennen, gar kein Zweifel zurück bleibt«. René Descartes: Regulae ad directionem ingenii. Regeln zur Ausrichtung der Erkenntniskraft, krit. revid., übers. u. hrsg. v. Heinrich Springmeyer, Lüder Gäbe, Hans Günter Zekl, Hamburg, 1973, Regel III, Abs. 8 und 5, S. 17-21.

20 Thomas Hobbes: Leviathan, Frankfurt a.M. 1994, S. 36. 


\section{Deutungskämpfe I: Naturwissenschaften versus Geisteswissenschaften - Erklären gegen Verstehen}

Die Standardversion der Geschichte dieser Zweiteilung der Wissenschaften sieht so aus: Die Naturwissenschaften hatten sich mit ihren Forschungen seit Kepler, Galilei und Newton bereits als Wissenschaften etabliert. Als dann im 19. Jahrhundert Sprach-, Geschichts- und Gesellschaftswissenschaften auf den Plan traten und sich ebenfalls als Wissenschaften zu behaupten suchten, lehnten sie das Erklärungsmodell der Naturwissenschaften mit seinen Ableitungen aus allgemeinen Naturgesetzen ab und konturierten eine neue wissenschaftliche Methode, nämlich das Verstehen. ${ }^{21}$

An solchen geläufigen Erzählungen ist viel Wahres, doch meist genügen sie nicht, um begreiflich zu machen, wo der entscheidende Punkt liegt. Wenn das Motiv des Erklärens - soweit wir bisher sehen - im Beweisen, also in der zwingenden Ableitung aus allgemeinen Prinzipien und Gesetzen besteht, worin besteht dann das Motiv des Verstehens? Was macht Verstehen aus? Bei der Charakterisierung kommen schnell wertende Vokabeln ins Spiel: naturwissenschaftliches Erklären gilt als >exakt<, geisteswissenschaftliches Verstehen als >vage‘; im Erklären gehe es rational zu, Verstehen habe dagegen mit Einfühlung zu tun. Solche Wertungen verknüpfen sich mit der Frage, ob Verstehen überhaupt als wissenschaftliches Verfahren gelten kann.

Im Jahr 1862 stellte Johann Gustav Droysen, einer der bedeutenden Historiker des 19. Jahrhunderts, fest:

»wenn es eine Wissenschaft der Ceschichte geben soll, [muss] diese ihre eigene Erkenntnisart, ihren eigenen Erkenntnisbereich haben [...]; und glücklicherweise gibt es zwischen Himmel und Erde Dinge, die [...] nicht erklärt, sondern verstanden werden wollen. $\ll^{22}$

21 Vgl. zu einer Darstellung aus der Perspektive philosophischer Wissenschaftstheorie: Georg H. von Wright: Erklären und Verstehen, Frankfurt a.M. 1993 (3. Aufl.).

22 Johann Gustav Droysen: Historik. Rekonstruktion der ersten vollständigen Fassung der Vorlesungen (1857), Grundriß der Historik in der ersten handschriftlichen (1857/1858) und in der letzten gedruckten Fassung. Textausgabe v. Peter Leyh, Stuttgart/Bad Cannstatt 1977, Beilage: >Erhebung der Ceschichte zum Rang einer Wissenschaft<, S. 451-469, hier S. 461. 
Jede Wissenschaft sei »eine Betrachtungsweise ${ }^{23}$ Die Physik betrachte ihren Gegenstand als »das im Wechsel Gleiche [...]: die Regel, das Gesetz, den Stoff, die Raumerfüllung « ${ }^{24}$; die Geschichtswissenschaft dagegen das »im Gleichen Wechselnde« - zwei Weisen der Betrachtung.

Gut dreißig Jahre später, in seiner Rektoratsrede Geschichte und Naturwissenschaft von 1894, wird Wilhelm Windelband Droysens Überlegungen aufnehmen und mit den Begriffen nomothetisch und idiographisch ebenfalls zwei nicht gegenstandsbedingt, sondern methodisch verschiedene wissenschaftliche Verfahrensweisen unterscheiden. Der Unterschied ist ein Unterschied der Richtung: während in den Naturwissenschaften »das einzelne gegebene Objekt « nur als »Typus, als Spezialfall eines Gattungsbegriffs [...] zur Einsicht in eine gesetzmäßige Allgemeinheit« relevant sei, betrachte die Geschichtswissenschaft »ein Gebilde der Vergangenheit in seiner ganzen individuellen Ausprägung «. ${ }^{25}$ Wie Droysen sieht Windelband den Unterschied der beiden Typen von Wissenschaft nicht als sachlichen, sondern als einen Unterschied in der Sicht- und Herangehensweise:

»Es bleibt möglich und zeigt sich in der Tat, dass dieselben Gegenstände zum Objekt einer nomothetischen und daneben auch einer idiographischen Untersuchung gemacht werden können. Das hängt damit zusammen, dass der Gegensatz des Immergleichen und des Einmaligen in gewissem Betracht relativ ist. Was innerhalb sehr grosser Zeiträume keine unmittelbar merklichen Veränderungen erleidet und deshalb auf seine unveränderlichen Formen hin nomothetisch behandelt werden darf, kann sich darum doch vor einem weiteren Ausblick als etwas nur für einen immerhin begrenzten Zeitraum Cültiges, d.h. als etwas Einmaliges erweisen. ${ }^{26}$

Beispiele, die Windelband anführt, sind Formgesetze von Sprachen, die Physiologie, Formationen der Geologie und die »Entwicklungsgeschichte« der Lebewesen. ${ }^{27}$ Damit wird die Pointe Windelbands klar: Ein wissenschaft-

23 Droysen: Historik. Beilage `Natur und Geschichte〈, S. 470-479, hier S. 477.

24 Droysen: Historik. Beilage `Natur und Geschichte`, S. 470-479, hier S. 474.

25 Wilhelm Windelband: Ceschichte und Naturwissenschaft, Straßburger Rektoratsrede 1894, Straßburg 1904, S. 16.

26 Windelband: Ceschichte und Naturwissenschaft, S. 12.

27 Vgl. Windelband: Geschichte und Naturwissenschaft, S. $12 f$. 
liches Phänomen, wie z.B. die Entstehung des Lebens, kann entweder als historisches Ereignis aufgefasst und beschrieben werden - man versucht dann diesen Prozess, wie er sich tatsächlich zugetragen hat, möglichst detailliert und umfassend zu rekonstruieren -, oder aber als gesetzmäßiger Zusammenhang erklärt werden - was bedeutet, die Bedingungen zu formulieren, unter denen Leben immer und auf die gleiche Weise entstehen würde. Es ist eine Frage des Erkenntnisinteresses, der Blickrichtung, der inneren Ausrichtung und Struktur - damit scheint der Gedanken auf, dass es Unterschiede der Typen von Wissenschaft gibt.

Doch, weit davon entfernt, eine Gleichwertigkeit unterschiedlicher Typen $\mathrm{zu}$ akzeptieren, blieb die Idee der Wissenschaft ein umkämpftes Feld. Zunächst hat sich der englische Sprachgebrauch seit Mitte des 19. Jahrhunderts mehr und mehr auf eine Seite gestellt: Science hießen zumeist nur noch die Naturwissenschaften vom Typ Physik, alle anderen Disziplinen fielen unter $\mathrm{Hu}$ manities. ${ }^{28}$ Und diese Verengung des Begriffs hatte weitreichende Folgen. In England nämlich ging es seitdem nicht um das Verhältnis von zwei Arten von Wissenschaft, es ging nicht um ein Verhältnis auf Augenhöhe, es ging vielmehr um einen Kampf zwischen zwei kulturellen Idealen: dem der naturwissenschaftlich-technischen und dem der klassisch-humanistischen Erziehung.

Nicht zwei Arten von Wissenschaften, sondern zwei Kulturen prallten hier aufeinander - und dies war, als C. P. Snow 1959 den Begriff von den Two Cultures prägte, längst vorbereitet. Bis Mitte des 19. Jahrhunderts nämlich war die klassische Bildung als "proper education of a gentleman ${ }^{29}$ an den englischen Universitäten allein bestimmend. Erst 1850 wagte Cambridge, einen Kurs in natural science anzubieten. 1880 forderte dann Thomas H. Huxley, ein Biologe in den Fußstapfen von Darwin und Hume, das klassizistische Bildungsideal heraus und klagte eine Anerkennung der Naturwissenschaften in ihrem geistigen Wert und in ihrem Beitrag zum nationalen Wohlstand ein. ${ }^{30}$ Wie im Fall Snows einige Jahrzehnte später folgte prompt eine Antwort von Seiten der eta-

28 Zur Geschichte des Gegensatzes von Science und Humanities vgl. Benjamin R. Cohen: Science and humanities: across two cultures and into science studies. In: Endeaver, 1(2001), S. 8-12.

29 Stefan Collini: Introduction. In: C. P. Snow: The two Cultures, Cambridge University Press 1998, S. vii-Ixxii, hier S. xiii

30 vgl. Collini, 1998, S. xiv. 
blierten Bildung: Der »leading man of letters in Victorian England $\aleph^{31}$, Matthew Arnold, konzedierte, dass beide, Wissenschaft und klassische Bildung, ihren Platz haben sollten, dass aber, zur Heranbildung eines »educated man $\aleph^{32}$ das Studium der Literatur, insbesondere der antiken Literatur unverzichtbar sei.

Damit war der Rahmen vorgezeichnet für den Einsatz C. P. Snows, der in seinem scharfen Plädoyer eben nicht zwei Typen der Wissenschaft einander gegenüberstellte, sondern zwei Kulturen, von denen eine das neue Selbstbewusstsein der modernen Naturwissenschaften repräsentierte, die andere die untergehende Welt der gesellschaftlichen Eliten mit ihren traditionellen Bindungen und ihren aristokratischen und großbürgerlichen Bildungsidealen.

Und dieser Unterschied zwischen Science einerseits und gehobener Bildung andererseits liegt auch noch den Debatten um Wissenschaft zugrunde, die in den 1990er Jahren unter dem Titel Science Wars zuerst in den USA geführt, dann auch international zum Thema wurden. Zwar spielen hier auch andere Motive eine Rolle - nämlich die Weise, in der Naturwissenschaften in der Perspektive postmodernen Denkens verstanden wurden (Stichwort: Konstruktivismus - dazu später mehr) - doch ging es noch immer um die Kluft zwischen Science einerseits und anderen intellektuellen Feldern andererseits. Natürlich hatten sich die Machtverhältnisse inzwischen nicht nur in den USA gründlich gewandelt. Als in Deutschland das Bundesministerium für Bildung und Forschung 2007 ein गJahr der Geisteswissenschaften< ausrief, waren dem ein \Jahr der Physik (2000), eines der Lebenswissenschaften (2001), der Geowissenschaften (2002), der Chemie (2003) der Technik (2004), wieder der Physik (2005) und der Informatik (2006) vorangegangen, das Jahr der Mathematik folgte; Geisteswissenschaften als ein einziger großer Topf und unter ferner liefen, wobei Sozialwissenschaften ganz fehlten.

Muss man also sagen, dass die Konkurrenz zwischen Erklären und Verstehen nur eine Episode war? Dass Wissenschaft nun weltweit zu natural science zusammengeschmolzen ist? Ein Blick in die Realität der Universitäten und Forschungseinrichtungen zeigt ein völlig anderes Bild: geistes- und sozialwissenschaftliche Disziplinen und Studiengänge florieren neben naturwissenschaftlichen in großer Breite und Intensität. Ist es also nur eine merkwürdige Prägung des Blicks, eventuell bestimmt durch die angelsäch-

31 Collini, 1998, S. xiv

32 Collini, 1998, S. xiv 
sische Situation und Debatte, die dieses Faktum so leicht vergessen und die Verknüpfung so geläufig macht, dass sie sich fast automatisch einstellt: wer Wissenschaft denkt, denkt - zuerst und zumeist - Naturwissenschaft. Was ist zu tun, um diesen heimlichen Automatismus aufzubrechen? Jedenfalls ist es nötig, sich die Seite des Verstehens gründlicher anzusehen, zu begreifen, wie sie in sich strukturiert ist, wie sie sich im 19. Jahrhundert als alternative Wissenschaftsform konstituierte und wie sie zu dem steht, was sich im 12 . und 13. Jahrhundert als scientia herausschält.

\section{Quellen moderner Wissenschaften II: Die Tradition von Hermeneutik und Interpretation}

Als sich zu Beginn des 13. Jahrhunderts in Paris, Bologna, Oxford und anderen Städten Universitäten zu formieren begannen und sich durch die Rezeption der Zweiten Analytik ein neuer Begriff von Wissenschaft als Beweisführung ausprägte, war das Feld, in dem dies stattfand, natürlich schon bestellt. Denn Erkenntnis, auf die es ankommt, war Erkenntnis im Modus von Textverstehen, von Hermeneutik und Interpretation.

Auch hier vordergründig eine erstaunliche Kontinuität: Sowohl das griechische Verb hermeneuein als auch das lateinische Verb interpretari bezeichnen übersetzen, deuten, vermitteln, auslegen. Während die griechische Vokabel theoretisch marginal blieb, ${ }^{33}$ wurde sowohl in der lateinischen Philologie und Rhetorik als auch insbesondere in der Jurisprudenz das Verb interpretari mitsamt seinen Ableitungen interpres und interpretatio »seit Plautus [254-184] ständiger Besitz der gesamten lateinischen Literatur «. ${ }^{34}$ Des Weiteren wurde die aristotelische Schrift Peri Hermeneias (dt. Lehre vom Satz) ${ }^{35}$ ins Lateinische mit De In-

33 Hans-Georg Gadamer führt in seinem Artikel Hermeneutik (Historisches Wörterbuch der Philosophie, hrsg. v. Joachim Ritter, Bd. 3, S. 1062-1073) nur wenige verstreute Textstellen auf.

34 Manfred Fuhrmann: Interpretatio. Notizen zur Wortgeschichte. In: Sympotica Franz Wieacker, Cöttingen 1970, S. 80-110, hier S. 81.

$35 \mathrm{Ob}$ der Titel von Aristoteles stammt, ist umstritten, auch der Zusammenhang zur Themenstellung der Interpretation ist nur, aber immerhin, indirekt zu rekonstruieren. Vgl. Meinrad Böhl/Wolfgang Reinhard/PeterWalter (Hrsg.): Hermeneutik: Die Ceschichte der abendländischen Textauslegung von der Antike bis zur Cegenwart, Wien u.a. 2013, S. 14-15. 
terpretatione übersetzt, ebenso die Vokabel hermeneia aus der Paulus Textstelle 1. Korinther 12,10 in der Vulgata (um 400 n. Chr.) mit interpretatio. ${ }^{36}$

Gegenstände der Praxis interpretari sind stets Texte oder textförmig gedachte Strukturen, die der besonderen Vermittlung bedürfen. Dies wird in zahllosen Belegstellen deutlich, z.B. wenn Cicero erklärt, dass für das wahre, naturrechtliche Gesetz »nicht nach einem Erklärer oder Ausleger [explanator aut interpres] « gesucht werden müsse ${ }^{37}$ oder, etwa 600 Jahre später, wenn in den von Iustitian in Auftrag gegebenen Institutionen (entworfen als Einführung und Lehrbuch des römischen Rechts) zu lesen ist: »Von Alters her war es nämlich so eingerichtet, dass es Männer gab, die das Recht öffentlich auslegen sollten [publice interpretarentur] «. ${ }^{38}$ Diese Longue-Durée-Kontinuität, die sich beliebig über Bacon in die Neuzeit mit Belegstellen verlängern ließe, ${ }^{39}$ zeigt, dass eine bestimmte Konzeptualisierung von Erkenntnis nicht erst in der modernen Bibelauslegung und Philologie, sondern schon mit der Rezeption insbesondere der lateinischen Juristik und Rhetorik sich als eine eigenständige Könnerschaft, eine ars etabliert hatte.

\section{Verstehen als Arbeit an sich selbst und den eigenen Konzepten}

Für die Tradition der christlichen Hermeneutik spielte De Doctrina Christiana, ein Werk, das Augustinus um 400 n. Chr. verfasste, eine entscheidende Rolle. ${ }^{40}$ Der Titelbegriff doctrina ist nicht leicht zu übersetzen, da es in dem Werk weniger um eine Lehre im Sinne einer Doktrin, denn um eine methodische Reflexion geht. ${ }^{41}$ Diese Methode setzt Augustinus explizit ins Verhältnis zu scientia, die er als eine bestimmte Stufe in den Erkenntnisweg der doctrina

36 Vgl. Gerhard Kurz: Hermeneutische Künste. Die Praxis der Interpretation, Stuttgart 2018, S. 7.

37 Marcus Tullius Cicero: De re publica. Vom Staat. Übers.u. hrsg. v. Michael von Albrecht, Stuttgart 2013, 3. Buch, Kap. 22; S. 201.

38 Corpus luris Civilis. Die Institutionen, hrsg. v. Rolf Knütel, Berthold Kupisch, Sebastian Lohsse u. Thomas Rüfner, Heidelberg u.a. 2013 (4. Aufl.), Erstes Buch, Zweiter Titel, 8, S. 4.

39 Vgl. hierzu: Kurz: Hermeneutische Künste 2018; Böhl u.a.: Hermeneutik 2013.

40 Zur Rolle von Augustinus in der Hermeneutik vgl. Böhl u.a.: Hermeneutik 2013, S. 162-164.

41 Karla Pollmann: Nachwort. In: Aurelius Augustinus: Die christliche Bildung (De doctrina christiana). Übersetzung, Anmerkung und Nachwort v. Karla Pollmann, Stuttgart 2013, S. $260 f$. 


\section{BASISWISSEN 2: Hermeneutik - verstehende Wissenschaft}

Der Begriff Hermeneutik wurde im 17. Jahrhundert eingeführt zur Bezeichnung eine Methoden- oder Kunstlehre des Verstehens (Erster Titel: Hermeneutica Sacra sive Methodus exponendarum S. Literarum, Dannhauer 1654). Mit >Kunst $<$ ist hier im Anschluss an das griechische techné und das lateinische ars eine Könnerschaft gemeint, die eine innere Systematik aufweist, somit lehr- und lernbar ist. Im Anschluss an diesen Impuls entwickelte sich eine umfassende Tradition der Verstehenslehren, die mit dem Begriff der Hermeneutik assoziiert war. Sie bündelte die Traditionsstränge der antiken und mittelalterlichen Interpretationslehren wie sie in der Philosophie, Philologie, Juristik und Rhetorik bereits vorhanden waren.

Begrifflich und systematisch nahm sie dabei ein Grundmotiv aus der aristotelischen Schrift Peri Hermeneias in Gebrauch, nämlich das Problem der Übermittlung und des Nachvollzugs eines ursprünglichen Satzsinns. Als eine Bewegung der Systematisierung und Verwissenschaftlichung prägte sie schon früh die hermeneutischen Grundmotive: die Interpretation aus der Beziehung von Teil und Ganzem, die sich anpassende, nachahmenden Rekonstruktion und die kritische Reflexion, auch des eigenen Standpunkts.

Im 19. Jahrhundert wurde die philologische Hermeneutik (Schleiermacher) und die juristische Hermeneutik (Savigny) systematisch ausgebaut, daneben entstand eine hermeneutische, interpretative Geschichtswissenschaft (Droysen); im 20. Jahrhundert eine ,Verstehende Soziologie`(Weber) und >Hermeneutische Ethnologie، (Geertz). Die >Philosophische Hermeneutik^ (Heidegger, Gadamer, Ricoeur, Taylor) entfaltet eine Interpretation des menschlichen Weltverhältnisses als eines, das von Grund auf durch Verstehen konstituiert wird. In ihrer Weiterentwicklung wurden auch das Nicht-Verstehen und die Grenzen des Verstehens zu zentralen Themen.

integriert. Unter scientia fallen sämtliche Wissensdisziplinen der Antike wie Geschichtsschreibung, Astronomie, Dialektik und Mathematik. Doch keine 
dieser Disziplinen ist für Augustinus Selbstzweck, sie stellen lediglich Kontextwissen zum Verständnis der Heiligen Schrift bereit.

Das antike Motiv der Deutungsbedürftigkeit von Texten wird in der christlichen Tradition so transformiert, dass deren Interpretationsbedürftigkeit auf eine Steigerung des Erkenntnisbemühens hin angelegt ist. Augustinus beantwortet die Frage danach, warum Gottes Rede mit dunklen Stellen und Allegorien versetzt sei, so:

»Die nämlich nicht geradewegs finden, was sie suchen, mühen sich unter Hunger ab. [...] Denn prächtig und heilsam hat der HI. Geist die HI. Schrift so umgeformt, dass er mit klareren Stellen dem Hunger begegnet, mit den dunkleren aber den Überdruß vertreibt. Fast nichts nämlich wird in jenen dunklen Stellen aufgestöbert, was nicht anderswo in klarster Weise ausgedrückt gefunden werden kann.« ${ }^{42}$

Gut tausend Jahre später, inzwischen hatte sich eine protestantische Verstehenslehre ausgebildet, handelt Matthias Flacius in ganz ähnlicher Weise von den »Ursachen der Schwierigkeit der Heiligen Schrift« und kommt zu dem Schluss:

»Vieles ist selbst den Frommen verborgen, damit sie um so eifriger die Heilige Schrift erforschen und eine klarere Offenbarung erstreben. [...] Schließlich sollen wir hier Gott und seine Mysterien wie in einem Rätsel und unvollkommen erkennen, im anderen Leben aber vollständig und von Angesicht zu Angesicht sehen. $^{43}$

»Wie in einem Rätsel«: Diese Formulierung bringt ein zentrales Motiv in der Tradition der Verstehenslehren zum Ausdruck. Denn wenn ein Erkenntnisgegenstand in rätselhafter Weise gegeben ist, dann erfordert dies nicht nur intensive, sich hineinversetzende Beschäftigung mit ihm, es erfordert auch eine bestimmte Forschungsweise: Während das Erklären im Sinne des Beweis-

42 Augustinus: Die christliche Bildung (Dedoctrina christiana) [426/427], Stuttgart 2002, Zweites Buch, VI.7.14, S. 51.

43 Matthias Flacius Illyricus: De ratione cognoscendi sacras literas. Über den Erkenntnisgrund der Heiligen Schrift, übers., eingel. u. m. Anm. vers. v. Lutz Geldsetzer, [es handelt sich bei dieser Textausgabe um den Anfang des zweiten Teils der Clavis scripturae sacris von 1567, vgl. das Vorwort des Herausgebers], Düsseldorf 1968, S. 23. 
schlusses so etwas wie eine geistige Maschine entwirft, in der sich das eine aus dem anderen zwangsläufig ergibt, bewegt sich das Rätsellösen im Gestus des Suchens. Stets muss nach Möglichkeiten gesucht werden, es muss arrangiert und probiert werden, bis sich eine Lösung zeigt. Friedrich Schleiermacher drückt die Zugangsweise des Verstehens in der Formulierung aus, dass wir

»öfter vom Ende zum Anfang zurückkehren und das Auffassen ergänzend von neuem beginnen; je schwieriger die Cliederung des Ganzen zu fassen ist, desto mehr suchend ihr vom Einzelnen aus auf die Spur [...] kommen, je reichhaltiger und bedeutsamer das Einzelne ist, um so mehr suchend es vermittelst des Ganzen in all seinen Beziehungen [auffassen]. ${ }^{44}$

Suchen heißt im Griechischen zetein. Um dieses Moment des Suchens im Verstehen herauszustellen, spricht man auch von einer "zetistischen «, einer "forschenden " Interpretation. ${ }^{45}$ Dieses suchende Moment sei nämlich gemeint, wenn win der hermeneutischen Literatur von der Erfahrung, dem Wissen, der Kongenialität, dem Takt und >Fingerspitzengefühl, von Intuition und Divinationsgabe des Hermeneuten gesprochen wird «. ${ }^{46}$ Das Konzept des Forschens stammt nicht aus den Beweislehren, in denen es ums Demonstrieren geht, es stammt aus den Verstehenslehren: denn die Worte research und forschen bezeichnen eine intellektuelle Haltung des Suchens und des Fragens, die dann auftaucht, wenn man wissen will, wie Dinge miteinander zusammenhängen, wie sich aus Teilen ein Gesamtzusammenhang ergibt oder woraus ein Zusammenhang besteht.

Vor diesem Hintergrund ist offensichtlich, welches Spannungsfeld sich eröffnete, als die Zweite Analytik zu versprechen schien, über die formale Korrektheit logischer Schlüsse hinaus einen Weg zu bieten, auch inhaltlich aus wahren Prinzipien auf wahre Folgerungen übergehen zu können. Für Augustinus bestand scientia (bestenfalls) in hilfreichen Kenntnissen, nun konnte es so scheinen, als ob in scientia selbst der Weg zur Wahrheit liege.

44 Friedrich Schleiermacher: Über den Begriff der Hermeneutik mit Bezug auf F.A. Wolfs Andeutungen und Asts Lehrbuch. In: Hermeneutik und Kritik, hrsg. u. eingel. v. Manfred Frank, S. 309-346, hier S. 333f.

45 Vgl. Lutz Geldsetzer: >Einleitung‘zu Matthias Flacius Illyricus: De ratione cognoscendi sacras literas. Über den Erkenntnisgrund der Heiligen Schrift, Düsseldorf 1968, S. XIVff.

46 Geldsetzer: >Einleitung〈1968, S. XV. 
Genauer: als ob es eine intellektuelle Technik gebe, die, richtig angewandt, in gleichsam mechanisch-zwingender Weise Wahrheit freilegt.

Für das Verhältnis von Erklären und Verstehen ist der Kontrast zwischen einer scientia, die als Technik Wahrheiten demonstriert und einer sapientia, die als Einsicht aus einem Weg der Selbstveränderung und Selbstläuterung resultiert, von grundlegender Bedeutung. Denn es ist dieses Moment der Selbstveränderung des Erkennenden in der Erkenntnis, das als ein Grundmotiv der Tradition der Verstehenslehren in Rechnung zu stellen ist. Erkennen bedeutet hier, sich auf einen Weg zu machen, auf dem das System der eigenen Überzeugungen grundlegend verwandelt wird.

\section{Quellen moderner Wissenschaften III: Die Tradition der Naturalis historia - Beschreiben, Entdecken, Klassifizieren}

Mit dem Erklärungs- und dem Verstehensparadigma ist es nicht getan: Es gibt noch einen dritten Strang, in dem sich Ideen zu Wissenschaft gebündelt und tradiert haben. War das erste Modell charakterisiert durch die Verben beweisen, demonstrieren und erklären, das zweite durch verstehen, rätsellösen, rekonstruieren, so treffen wir nun auf eine dritte Familie von Vokabeln: beobachten, beschreiben, sammeln, zuordnen, klassifizieren. Wer beobachten und sammeln will, muss finden und unterwegs sein. Deshalb gehört zu diesem Thema der Begriff des Entdeckens und damit auch der Entdeckungsreise, der Forschungs- und Erkundungsfahrt, der Expedition. Dass Wissenschaft in erster Linie mit Entdeckungen und dem Sammeln und Ordnen von Fakten $\mathrm{zu}$ tun hat, diese Idee ist sicherlich nicht weniger tief verankert als diejenige, dass es bei Wissenschaft ums Beweisen oder Verstehen geht. Deshalb ist auch zu erwarten, dass sie ebenfalls über eine lange und ehrwürdige Tradition verfügt. Und tatsächlich finden wir hier einen kanonischen Text, der für die gesamte Traditionslinie namensgebend wurde: die Naturalis Histori$a e$, verfasst von Plinius Secundus (der Ältere), einem römischen Gelehrten in der Zeit bis 77 n. Chr. In 37 Bänden versammelt das Werk weite Bereiche des Wissens der Antike aus den Sachgebieten Kosmologie, Astronomie, Meteorologie, Geologie, Geographie, Anthropologie, Zoologie, Botanik, Pharmakologie, Mineralogie, Metallurgie, Plastik und Malerei. Grundlage der Abhandlungen sind tausende von Exzerpten von über 400 Autoren, die auch 


\section{BASISWISSEN 3: Historia - ein missverständliches Wort}

Mit unserem Wort Historie verbinden wir Gedanken an Geschichte im Sinne eines zeitlichen Ablaufs. Das griechische Wort historia bedeutet aber ganz allgemein das Erforschen und das durch Befragen und Beobachten Erkannte und damit auch die Kenntnis, die Kunde und auch der Bericht von etwas. Die Libros Naturalis Historiae des Gaius Plinius Secundus sind also als Naturgeschichte im Sinne einer $\mathrm{Zu}$ sammenstellung von Erkenntnissen der Naturforschung, im Sinne einer Naturkunde und ihrer Darstellung zu verstehen.

Anknüpfend an Plinius entfaltete sich seit dem 18. Jahrhundert ein Wissenschaftstypus, der in der Sammlung und Systematisierung von Naturphänomenen bestand, besonders der Botanik, Zoologie, Mineralogie, Medizin und Anthropologie. Die Begriffe Naturgeschichte, Histoire Naturelle und Natural History begegnen heute vielfach im Zusammenhang mit naturwissenschaftlichen Sammlungen und naturkundlichen Museen.

Eine verzeitlichende Verwendung des Begriffs findet sich prominent erst im 18. Jahrhundert, etwa in Kants Allgemeine Naturgeschichte und Theorie des Himmels (1755) - und zwar in der Programmatik, die >Ordnung der Natur durch >Auswicklung`, ausgehend von einem chaotischen Anfangszustand zu begreifen, somit: »sich ein wohlgeordnetes Ganze erzeugen zu sehen« (S. 232; A XXII; XXIII).

In der Folge konnte sich Naturgeschichte mit dem Konzept einer Evolution (>Auswicklung`) verbinden: Naturgeschichte ließ sich nun als Entwicklungsgeschichte des Weltalls, der Lebewesen, des Menschen und menschlicher Eigenschaften verstehen.

namentlich genannt werden. Auch eigene Kenntnisse, teils erworben auf weitläufigen Reisen, flossen ein - und Plinius bedauerte, dass die griechische Kultur der Forschungsreisen in Rom aus der Mode gekommen war. ${ }^{47}$

47 Vgl. Arno Borst: Das Buch der Naturgeschichte: Plinius und seine Leserim Zeitalter des Pergaments (Abhandlungen der Heidelberger Akademie der Wissenschaften/Philosophisch-historische Klasse), Heidelberg 1994, S. 19 u. 24. 
Das umfangreiche Werk wurde durch zahlreiche Abschriften das gesamte Mittelalter durch tradiert und blieb auch in der Neuzeit ein wichtiger Bezugspunkt naturwissenschaftlichen Wissens ${ }^{48}$ Allein zwischen 1469 und 1799 sollen 2222 komplette und 281 Auswahlausgaben $\star^{49}$ erschienen sein. Die Verbreitung des Werks begründete eine Tradition, die ihren Höhepunkt im 18. und frühen 19. Jahrhundert erreichte.

Am Anfang einer Kette großer und bis heute für die Wissenschaften wichtiger Namen steht Carl von Linné, der schwedische Gelehrte, der ab 1737 in den zwölf - jeweils ausgeweiteten - Auflagen seines Werk Systema Naturae schließlich etwa 7700 Pflanzen-, 6200 Tier- und 500 Mineralienarten beschrieb und klassifizierte. Parallel verfasste der französische Naturforscher Georges Louis Leclerc Buffon zwischen 1749 und 178836 Bände der (später noch ergänzten) Histoire naturelle générale et particulière. 1755 erschien Immanuel Kants Allgemeine Naturgeschichte und Theorie des Himmels und 1790 machte sich Alexander von Humboldt zunächst mit dem Naturforscher und Weltreisenden Georg Forster auf zu einer Forschungsreise an den Niederrhein, dann zu einer großangelegten Amerikareise mit verschiedenen Expeditionen zwischen 1799-1804, die schließlich in das 1845-1862 publizierte Werk Kosmos - Entwurfeiner physischen Weltbeschreibung mündete.

Diese Epoche des Reisens und Beschreibens war zugleich eine der großen Sammlungen. Gesteine, Pflanzen, Insekten, Muscheln, Geräte, anatomische Präparate: nicht nur der Adel repräsentierte sich durch große Kunst- und Naturalienkabinette, sondern auch das Bürgertum entwickelte eine Leidenschaft, Sammlungen aller Art anzulegen. ${ }^{50}$ Die Rolle, die die Sammlungen für die Wissenschaft spielten, lässt sich wiederum an Linné belegen: Sein flexibler Herbarschrank, der so angelegt war, dass seine Fächer nicht fix waren, sondern nach Bedarf eingeteilt und ergänzt werden konnten, kann als ein Werkzeug der Systematisierung betrachtet werden. So zeigt sich in diesem Möbelstück »eine neue empirische Wissenschaft, die sich auf Beob-

48 Vgl. Gerhard Winkler: Anhang; Naturalis Historia, in: Plinius Secundus, Gaius: Naturkunde (lateinisch - deutsch), hrsg. u. übers. v. Roderich König in Zus. m. Joachim Hopp u. Wolfgang Glöckner, Düsseldorf/Zürich 1997 (2. Aufl.), S. 353-385, hier S. 373.

49 Winkler: Anhang; Naturalis Historia, S. 373.

50 Vgl. Henning Bock: BürgerlicheSammlungen im18. Jahrhundert. Bildungdurch Anschauung. In: Annemarie Gethmann-Siefert/Elisabeth Weisser-Lohmann/Bernadette CollenbergPlotnikov (Hrsg.): Kunst als Kulturgut. 2, »Kunst«und»Staat«., München 2011, S. 33-50. 
achten und Beschreiben, Sammeln und Klassifizieren stützte und die damit im 18. Jahrhundert die Grundlage der modernen Systematik auf Grund von Fakten und Objekten legte. $\aleph^{51}$

Naturgeschichte, Histoire naturelle, Natural History: solche Titel lassen an längst vergangene Wissenswelten denken. Aber tatsächlich ist das Erheben von Daten und ihre Integration in Ordnungssystemen (Taxonomien) nach wie vor ein Kernbereich wissenschaftlicher Tätigkeit. Wenn es in der biologischen Systematik z.B. heute darum geht, mittels DNA-Analysen Verwandtschaftsbeziehungen zwischen Pflanzen- und Tierarten nachzuzeichnen, dann geschieht hier, was die Aufgabenstellung anbelangt, etwas sehr Ähnliches wie in den Hochzeiten der Naturgeschichte. Einblick und Überblick über die Ordnung der Dinge - so der deutschsprachige Titel eines Werks von Michel Foucault, in dem er das taxonomische Denken des 18. Jahrhunderts analysierte ${ }^{52}-$ zu gewinnen, ist nach wie vor das Bestreben dieses Typs wissenschaftlicher Arbeit. Schon die Naturalis Historiae ist keine bloße Zusammenstellung von Wissen, sondern folgt einer inneren Systematik der Gegenstände. ${ }^{53} \mathrm{Zu}$ den frühen Klassifikationssystemen der belebten und unbelebten Natur sind inzwischen eine Vielzahl neuer Systematiken hinzugekommen: Sprachen, chemische Elemente, Krankheiten, literarische Texte, Ethnien, Artefakte - für alle diese Sachen gibt es Einteilungen und tabellarische Gliederungen, die Ordnungssysteme darstellen.

Als mediale Form erlebte die Anordnung von "Information in tabellarischer Disposition $\aleph^{54}$ im 18. Jahrhundert ihre Blüte. Mit dem Anwachsen von Daten - 1623 habe man, so wird berichtet, 6000 botanische Arten, 1694 dann schon 10146 und nur zehn Jahre später 18655 gezählt ${ }^{55}$ - kommt allerdings auch die tabellarische Darstellung in vielen Bereichen an ihre Grenzen. Das gab Anlass, von einem »Ende der Naturgeschichte« im 19. Jahrhundert zu sprechen. ${ }^{56}$

51 Bock 2011, hier S. 33.

52 Michel Foucault: Die Ordnung der Dinge. Eine Archäologie der Humanwissenschaften [Les mots et les choses], Frankfurt a.M. 1971 [1966].

53 Vgl. Eugenia Lao: Taxonomic Organization in Pliny's Natural History. In: Papers of the Langford Latin Seminar, 16. (2016), S. 209-246.

54 ArndtBrendecke:Information intabellarischer Disposition. In: FrankGrunert/AnetteSyndikus (Hrsg.): Wissensspeicher der Frühen Neuzeit: Formen und Funktionen, Berlin 2015, S. 43-59.

55 Wolf Lepenies: Das Ende der Naturgeschichte: Wandel kultureller Selbstverständlichkeiten in den Wissenschaften des 18. und 19. Jahrhunderts, München 1976, S. 55.

56 Wolf Lepenies: Das Ende der Naturgeschichte München 1976. 
Doch was hier zu Ende ging, war lediglich die Idee, dass sich die Ordnung der Natur, zusammengefasst in einem umfassenden Werk, überblickshaft als Ganzes darstellen lässt. Was dagegen seit dem 17. Jahrhundert immer deutlicher Kontur gewann, war die Methode, möglichst umfangreiche Daten zu erheben und aus der Organisation dieser Daten Erkenntnisse zu gewinnen. Natürlich kam hier seit dem 19. Jahrhundert auch die Statistik ins Spiel. Zunächst war Statistik nur die Aufzeichnung von verwaltungstechnisch relevanten Daten wie Bevölkerungszahl, Geburten und Sterbefälle gewesen. Doch mit dem Einsatz mathematischer Verfahren entwickelte sich daraus ein methodisches Arsenal, das tief in unterschiedliche Wissenschaften hineinwirkte und auch darauf zielte, in Datenmaterial Regelmäßigkeiten aufzufinden. Auf das Verhältnis von Statistik und Wissenschaft kommen wir später noch ausführlicher zu sprechen.

Fassen wir zusammen: In der Traditionslinie der Naturgeschichte zeichnet sich ein Bild von Wissenschaft ab, das von den Konzeptionen der Scientia und der Verstehenslehren deutlich unterschieden ist. Wissenschaft als Erhebung und Darstellung von Faktenwissen ist offensichtlich eine ganz andere Vorstellung als die eines Beweisens, das durch Ableitung aus Prinzipien zustande kommt, oder die eines Verstehens, das sich in einen Prozess des suchenden Verknüpfens und Rätsellösens involviert.

Alle drei Bilder von Wissenschaft haben tiefe Wurzeln in der abendländischen Geschichte des Denkens und ebenso offensichtlich tragen alle drei nach wie vor Früchte. Müssen wir also von drei Gattungen von Wissenschaft sprechen? Die durch unüberschreitbare Schranken voneinander getrennt sind? Doch wie sollte sich diese Dreiteilung äußern? Offensichtlich lässt sich ja das Spektrum der gegenwärtigen wissenschaftlicher Disziplinen kaum in dieses Raster fügen. Vielleicht müssen wir noch einen Schritt weiter gehen, und unter den Oberflächen dieser Traditionen den Motiven und Methoden nachspüren, die die wissenschaftliche Praxis noch konkreter prägen. Was hat es mit der Mathematisierung als Kennzeichen von Wissenschaft auf sich? Was mit dem Experiment? Was bedeutet Analyse und Synthese? Was Induktion und Deduktion? Wohin gehört das Rekonstruieren und wohin das Modellieren? Welche Rolle spielt das Interpretieren? Welche die Kritik? Es ist Zeit, hier Klarheit und Orientierung zu finden - und dazu bietet das Gerüst der drei Wissenschaftstraditionen eine gute Voraussetzung. 